\title{
Efeitos de diferentes fontes de radiação na fisiologia e vida útil pós- colheita de camu-camu
}

\author{
Alex Guimarães Sanches ${ }^{1}$, Maryelle Barros da Silva ${ }^{1}$, Elaine Gleice Silva Moreira ${ }^{1}$, Jaqueline \\ Macedo Costa ${ }^{1}$, Carlos Alberto Martins Cordeiro \\ ${ }^{1}$ Universidade Federal do Pará, Campus Altamira, Altamira, Pará, Brasil. E-mail: alexsanches.eng@gmail.com, \\ maryellebarros@bol.com.br, elaine.moreira230@gmail.com,jackmacedo@gmail.com \\ ${ }^{2}$ Universidade Federal do Pará, Campus Bragança, Bragança, Pará, Brasil. E-mail: cancordeiro2006@ufpa.br
}

Recebido: 23/07/2016; Aceito: 17/05/2017

\section{RESUMO}

Por se tratar de um fruto climatérico, o camu-camu (Myrciaria dúbia (H.B.K.) McVaugh) apresenta uma rápida deterioração em temperatura ambiente, tal fato é atribuído à elevada taxa respiratória do fruto, ao aumento da produção de etileno e perda de água. Assim, o presente trabalho tem por objetivo avaliar os efeitos da irradiação gama e ultravioleta na sua conservação in natura. Os frutos foram mantidos sob refrigeração por um período de 21 dias em DIC fatorial composto por cinco tratamentos e oito tempos de avaliação. A cada três dias os frutos foram avaliados quanto à: perda de massa fresca, firmeza, sólidos solúveis, acidez titulável, pH, vitamina $\mathrm{C}$, carotenóides e taxa respiratória. As fontes de irradiação não diferiram estatisticamente ao longo do armazenamento, contudo, as dosagens avaliadas mostraram variação de modo que a utilização de $1,0 \mathrm{KGy}$ e kJ/m² de irradiação reduziu perda de massa e a perda de firmeza nos frutos, em contrapartida a dose de 2,0 KGy e $\mathrm{kJ} / \mathrm{m}^{2}$ manteve estáveis as características químicas garantindo uma vida de prateleira maior aos frutos de camu-camu.

Palavras-chave: Myrciaria dúbia (H.B.K.) McVaugh), análises físico-químicas, maturação vegetal

\section{Effects of radiation sources in physiology and life camu-camu post-harvest}

\begin{abstract}
Because it is a climacteric fruit camu-camu (Myrciaria dubia (HBK) McVaugh) shows a rapid deterioration in room temperature and attributed the high respiratory rate increased ethylene production and water loss. Thus, this study aims to evaluate the effects of gamma radiation and ultraviolet in its conservation in nature. The fruit were kept under refrigeration for a period of 21 days factor in DIC treatments consisting of five to eight times of evaluation. Every three days the fruits were evaluated: loss of weight, firmness, soluble solids, titratable acidity, $\mathrm{pH}$, vitamin $\mathrm{C}$, carotenoids and respiratory rate. The radiation sources did not differ statistically during storage, however, the evaluated dosages showed variation so that the use of $1,0 \mathrm{KGy}$ e $\mathrm{kJ} / \mathrm{m}^{2}$ irradiation reduced the mass loss and loss of firmness of the fruits, on the other hand the dose of 2,0 KGy e $\mathrm{kJ} / \mathrm{m}^{2}$ remained stable chemical characteristics ensuring a greater shelf life to the fruit camu-camu.
\end{abstract}

Key-words: Myrciaria dúbia (HBK) McVaugh), physical and chemical analysis, plant maturation. 


\section{Introdução}

O camu-camu (Myrciaria dúbia (H.B.K.) McVaugh), pertencente à família Myrtaceae, é um arbusto originário da Amazônia Brasileira e Peruana. Em seu habitat natural à beira de rios e igarapés ou regiões permanentemente alagadas a parte inferior de seu caule permanece submersa. Estudos tem sido conduzidos no sentido de ampliar as áreas de cultivo, aumentando a produção, melhorar a qualidade póscolheita e a comercialização dos frutos (ZANATTA e MERCADANTE, 2007).

Por se tratar de um fruto climatérico, o camu-camu apresenta uma rápida deterioração em temperatura ambiente sendo este fato atribuído à elevada taxa respiratória, ao aumento da produção de etileno e a perda de água que juntamente contribuem para diminuir o período de conservação do fruto. Desta forma, o uso de uma tecnologia adequada para melhorar sua conservação é fundamental para favorecer a comercialização do fruto in natura uma vez que o comércio do fruto se dá na forma processada.

Uma técnica que auxilia a conservação de frutas e vegetais é a irradiação, que tem sido utilizada em diversos países. A exposição à baixas doses de radiação pode diminuir a velocidade de amadurecimento e envelhecimento de frutas e vegetais, aumentando, desta maneira, a vida de prateleira e, consequentemente, as possibilidades de comercialização e maximização dos lucros tanto por parte dos produtores como por parte dos comerciantes (FRANÇOSO et al., 2008).

A radiação gama tem se revelado eficiente em prolongar a vida comercial de frutos frescos por retardar os processos de amadurecimento e senescência, reduzindo o apodrecimento sem provocar alterações significativas em seu aspecto, sabor e qualidade nutritiva (CAMPOS et al., 2011).

A radiação ultravioleta é um processo comprovadamente seguro e tem sido avaliado em muitas aplicações. Em função da dose aplicada no alimento, observa-se melhoria na qualidade microbiológica do produto, resultando na redução de perdas no armazenamento e prolongamento da vida útil (ALAM KHAN e ABRAHEM, 2010).

Desta forma, o objetivo neste experimento foi testar os efeitos da radiação gama e ultravioleta em diferentes dosagens visando o aumento da vida de prateleira em frutos de camu-camu sob refrigeração

\section{Material e Métodos}

Utilizou-se neste trabalho frutos de camu-camu colhidos em estádio de maturação fisiológico na cor vermelho no Campus Experimental da Embrapa Amazônia Oriental, localizado no município de Altamira-PA. Os frutos foram acondicionados em caixas térmicas e transportados até o laboratório de Ecofisiologia da Faculdade de Ciências Biológicas da Universidade Federal do Pará, Campus Altamira onde foram selecionados quanto à cor, à ausência de defeitos, injúrias e ataque por pragas e doenças.

Ainda no laboratório os frutos foram imersos em solução contendo $3 \mathrm{mg}$. L-1 de hipoclorito de sódio por um período de 5 minutos, posteriormente foram dispostos em bancadas de alumínio e secos a temperatura ambiente. Os frutos foram divididos em cinco lotes e submetidos aos tratamentos com irradiação gama nas concentrações de 1,0 e 2,0 KGy e irradiação UV-C a 1,0 e 2,0 kJ/m2, além do lote que não recebeu irradiação sendo representados pelo tratamento controle.

Para aplicação da radiação gama os frutos foram submetidos à fonte de Cobalto-60 e o irradiador utilizado foi o GAMMABEAN 650.

Para a aplicação da irradiação UV-C, os frutos foram mantidos sob a influência da iluminação ultravioleta (Ecolum e 15w) sendo a leitura realizada por um radiômetro. A determinação da dosagem para ambas as fontes de irradiação foi obtida em função da variação do tempo de exposição do fruto à fonte irradiadora.

Após a aplicação ou não dos tratamentos, os frutos foram acondicionados em embalagens de polietileno tereftalato com tampa (PET) e mantidos sob refrigeração a temperatura de $10^{\circ} \mathrm{C}$, simulando a comercialização nos pontos de venda a varejo por um período de 21 dias. O delineamento experimental adotado foi inteiramente casualizado sob esquema fatorial $5 \times 8$, sendo cinco tratamentos (controle, 1,0 e 2,0 KGy de radiação gama e 1,0 e 2,0 kJ/m2 de radiação ultravioleta) e oito tempos de avaliação $(0,3,6,9,12$, 15, 18, 21 dias); cada tratamento foi composto por cinco repetições e cada bandeja apresentava aproximadamente $250 \mathrm{~g}$ do fruto.

A cada três dias, avaliou-se cada tratamento quanto à perda de massa fresca, determinada pela diferença entre o peso dos frutos no dia inicial e o peso obtido a cada intervalo de tempo com os resultados expressos em porcentagem. A firmeza da polpa foi feita através do teste de firmeza $(\mathrm{N})$ em 10 frutos de cada bandeja (100 $\mathrm{g}$ de fruto cada) com o auxílio de um penetrômetro manual (FT02) fazendo-se uma punção na região central do fruto (IAL, 2008).

O teor de sólidos solúveis foi avaliado através de análise refratométrica utilizando refratômetro de bancada e os resultados expressos em ${ }^{\circ}$ Brix. A acidez titulável foi mensurada através do método titulométrico com solução de hidróxido de sódio $(\mathrm{NaOH}) \quad 0,1 \mathrm{~N}$, usando como indicador a fenolftaleína e os resultados foram expressos em porcentagem do ácido ascórbico/100 g de polpa, conforme o descrito por Aoac (2007). 
$\mathrm{O}$ pH foi medido diretamente na polpa, logo após processamento, utilizando-se um potenciômetro (Mettler DL 12) com membrana de vidro, aferido com tampões de pH 7 e 4 (AOAC, 2007). O conteúdo de vitamina $\mathrm{C}$ das amostras foi determinado pelo método colorimétrico (IAL, 2008), e os resultados, expressos em mg de ácido ascórbico por $100 \mathrm{~g}$ de polpa.

Os carotenóides totais foram determinados pelo método de Higby (1962), utilizado álcool isopropílico e $2 \mathrm{~mL}$ de hexano para extração dos pigmentos. As leituras foram feitas a $450 \mathrm{~nm}$ e os resultados expressos em $\mathrm{mg} / 100 \mathrm{~g}$.

A respiração foi determinada pela liberação de $\mathrm{CO} 2$, de acordo com metodologia adaptada de Bleinroth et al., (1976), utilizando-se para isso solução de hidróxido de bário saturado e solução de hidróxido de potássio $0,1 \mathrm{~N}$ com a seguinte fórmula: $\mathrm{T} \mathrm{CO} 2=2,2(\mathrm{VoV} 1)$. 10/P.T . Onde: $\mathrm{T} \mathrm{CO} 2$ = taxa de respiração $(\mathrm{mLCO} 2 . \mathrm{Kg}-1 . \mathrm{h}-1)$; Vo = volume gasto de $\mathrm{HCl}$ para titulação de hidróxido de potássio - padrão antes da absorção de $\mathrm{CO} 2(\mathrm{~mL})$; $\mathrm{V} 1$ = volume gasto de $\mathrm{HCl}$ para titulação de hidróxido de potássio após a absorção de $\mathrm{CO} 2$ da respiração $(\mathrm{mL})$; $\mathrm{P}=$ peso dos frutos; $\mathrm{T}$ tempo da respiração; $2,2=$ inerente ao equivalente de $\mathrm{CO} 2$ (44/2), multiplicado pela concentração do ácido clorídrico; $10=$ ajuste para $\mathrm{o}$ total de hidróxido de potássio usado no experimento.

As comparações múltiplas entre as médias dos parâmetros estudados foram realizadas utilizando-se teste de Tukey ao nível de 5\% de probabilidade através do software estatístico Bioestat 5.3. O modelo de regressão polinomial foi realizado utilizando o programa Excel tendo como base a significância do teste F.

\section{Resultados e Discussão}

A avaliação da perda de massa fresca indicou que o controle apresentou as maiores médias diferindo significativamente dos tratamentos em que houve a influência da radiação. Entre os tipos de radiação não se constatou diferença estatística, tão pouco nas dosagens testadas, contudo, dosagens de 2,0 KGy e $\mathrm{kJ} / \mathrm{m}^{2}$ de radiação gama e ultravioleta, respectivamente apresentaram os menores valores (Figura 1).

Guimarães et al., (2013) avaliando as dosagens de 0,5; 1,0 e 2,0 KGy de irradiação gama na pós-colheita de framboesas notaram melhores resultados quando tratadas nas dosagens de 2,0 KGy. Fato também verificado por Daiuto et al., (2010) que observaram efeito positivo do aumento do tempo de exposição à radiação ultravioleta na vida de prateleira de abacates.

O tempo de armazenamento foi o único fator que diferiu, independente do tratamento utilizado, observando maiores variações principalmente a partir do nono dia $(4,33 \%)$ para os frutos do tratamento controle e décimo quinto dia para aqueles tratados com radiação $(5,95 \%)$. Ao final de 21 dias independente da fonte de radiação e da dose utilizada, os valores médios oscilaram entre $7,89 \%$ e $8,13 \%$ enquanto no controle a média verificada ultrapassou os $10 \%$ (Figura 1)

As alterações na firmeza dos frutos de camu-camu estão representadas na Figura 2. Observa-se que até o nono dia não houve diferença significativa entre os tratamentos. A partir do décimo segundo dia, nota-se uma redução mais acentuada nos frutos do tratamento controle chegando ao final de 21 dias de armazenamento a apresentar cerca de $80 \%$ na redução de firmeza. Essa diminuição dos valores da firmeza é consequência do avanço do processo natural de amadurecimento e senescência dos frutos.

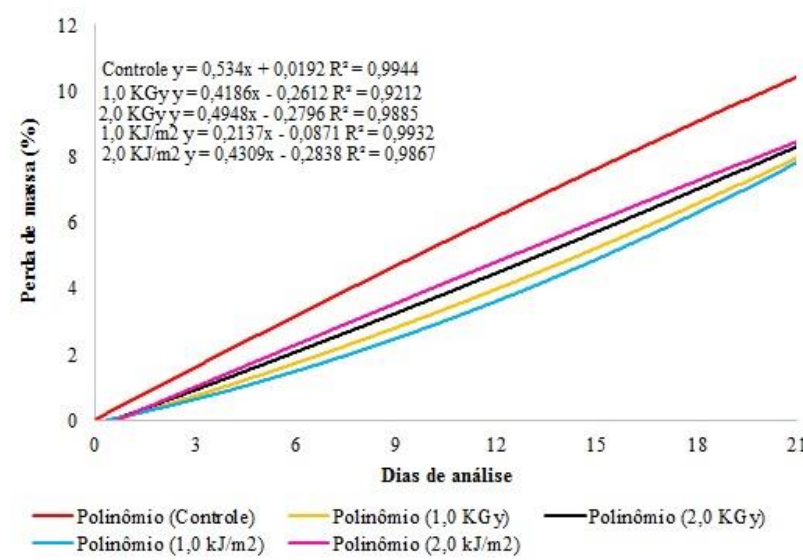

Figura 1. Perda de massa fresca (\%) em frutos de camu-camu tratados com diferentes fontes de radiação e armazenados sob refrigeração por 21 dias.

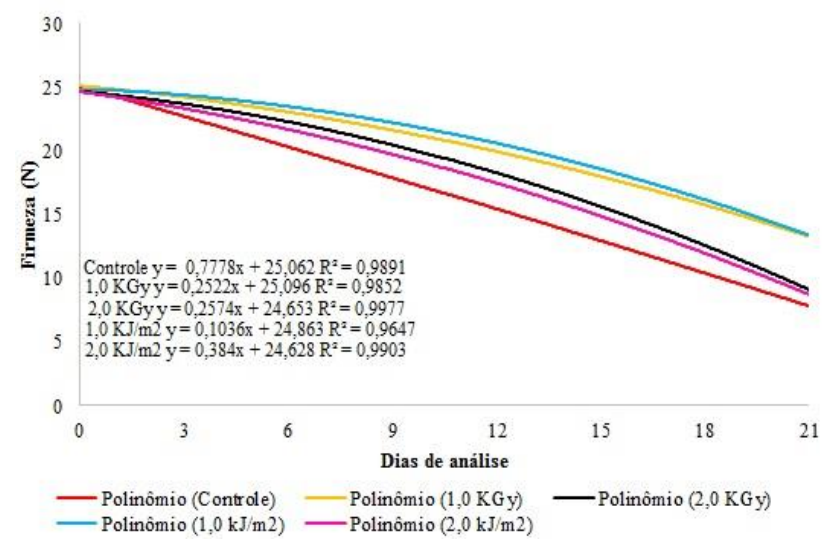

Figura 2. Redução nos valores de firmeza $(\mathrm{N})$ em frutos de camu-camu tratados com diferentes fontes de radiação e armazenados sob refrigeração por 21 dias.

Independentemente da fonte de irradiação utilizada, nota-se que os frutos tratados na dosagem mais alta $(2,0$ $\mathrm{KGy}$ e $\mathrm{kJ} / \mathrm{m}^{2}$ ) comprometeram os tecidos dos frutos reduzindo significativamente a firmeza a partir do décimo quinto dia e apresentando, ao final do 
armazenamento, média de $78,7 \%$, valor este semelhante à redução verificada nos frutos do tratamento controle cujo padrão de firmeza era proporcional ao estádio avançado de maturação dos frutos.

Fan et al., (2012) relatam que altas dosagens de irradiação podem provocar escurecimento, perda de firmeza, aparecimento de depressões superficiais, amadurecimento anormal e perda de sabor e aroma nos produtos considerando-se também, que cada produto tem uma determinada resposta específica à radiação UVC, dependendo da variedade e espécie. Semelhantes à estes resultados, Campos e Vieites (2010) e Guimarães et al., (2013) notaram menor firmeza em bagas de uvas 'Itália' e framboesas respectivamente, quando houve aumento na dosagem da irradiação.

Os tratamentos com a dosagem de $1,0 \mathrm{KGy}$ e $\mathrm{kJ} / \mathrm{m}^{2}$ apresentaram grande similaridade em seu armazenamento, podendo destacar a estabilidade da firmeza do fruto ao longo do armazenamento com percentual de redução de $53 \%$. O resultado apresentado implica que o consórcio da irradiação com a embalagem e refrigeração, propiciaram estabilidade suficiente da firmeza, para esses tratamentos, em 21 dias de armazenamento.

De acordo com a Tabela 1, observou-se uma oscilação nos valores de sólidos solúveis até o nono dia de armazenamento em todos os tratamentos, com posterior acréscimo até o último dia de armazenamento. Chitarra e Chitarra (2005) explicam que a diminuição nos valores de SS em alguns pontos ocorre devido à transformação do amido em açúcares e às condições da atmosfera no interior da embalagem.

Ainda de acordo com a Tabela 1, os tratamentos evidenciaram diferenças significativas entre si a partir do nono dia de avaliação; os tratamentos controle e com a dosagem de 2,0 KGy e $\mathrm{kJ} / \mathrm{m}^{2}$ de irradiação gama e ultravioleta apresentaram valores mais elevados, com média no fim do armazenamento de 8,37; 8,29 e 8,31 ${ }^{\circ}$ Brix, respectivamente. Os tratamentos com dosagem de $1,0 \mathrm{KGy}$ e $\mathrm{kJ} / \mathrm{m}^{2}$ de irradiação proporcionaram maior estabilidade nesses teores evidenciando médias ao fim de 21 dias de 7,35 KGy e 7,42 kJ/m² para radiação gama e ultravioleta respectivamente sem diferir entre si (Tabela 1). Tais resultados corroboram com a pesquisa de Oliveira et al. (2013), que evidenciaram diferença significativa nos teores de sólidos solúveis na polpa dos frutos de camu-camu sob influência da irradiação e temperatura de armazenamento.

De modo geral, os valores de sólidos solúveis encontrados neste experimento variaram de 4,17 a 8,37 ${ }^{\circ}$ Brix para todos os tratamentos estudados (Tabela 3). Estes resultados são concordantes com Silva et al. (2013) que obtiveram valores variando de 4,87 a 8,44 ${ }^{\circ}$ Brix para camu-camu colhidos no estádio de maturação 4. No camu-camu, os açúcares estão presentes em baixas concentrações, o teor de sólidos solúveis é representado por ácidos orgânicos que correspondem cerca de $40 \%$ de sua composição (ALVES et al., 2002).

Os valores referentes à acidez titulável nos frutos de camu-camu irradiados não apresentaram diferenças significativas entre si, verificou-se um aumento nesses valores em diferentes tempos de armazenamento com posterior redução até o fim do período experimental em todos os tratamentos (Tabela 2). Chitarra e Chitarra (2007) explicam que o aumento nos valores de acidez ocorre devido ao processo de maturação dos frutos e a redução é proveniente do consumo dos ácidos orgânicos que são metabolizados pela via respiratória e convertidos em moléculas não ácidas.

Tabela 1. Valores médios pelo teste de Tukey sobre o teor de sólidos solúveis ( ${ }^{\circ} \mathrm{Brix}$ ) em frutos de camu-camu tratados com diferentes fontes de radiação e armazenados sob refrigeração por 21 dias.

\begin{tabular}{cccccccccc}
\hline \multirow{2}{*}{ Tratamentos } & \multicolumn{7}{c}{ Dias de análise } \\
\cline { 2 - 8 } & 0 & 3 & 6 & 9 & 12 & 15 & 18 & 21 \\
\cline { 1 - 7 } Controle & $4,17 \mathrm{aA}$ & $5,21 \mathrm{abB}$ & $4,58 \mathrm{abB}$ & $5,37 \mathrm{abAB}$ & $6,64 \mathrm{bB}$ & $7,46 \mathrm{cB}$ & $8,05 \mathrm{cC}$ & $8,37 \mathrm{bC}$ \\
$1,0 \mathrm{KGy}$ & $4,17 \mathrm{aA}$ & $4,93 \mathrm{aB}$ & $4,28 \mathrm{aAB}$ & $5,12 \mathrm{aA}$ & $5,79 \mathrm{aA}$ & $6,47 \mathrm{aB}$ & $7,11 \mathrm{aB}$ & $7,35 \mathrm{aB}$ \\
$2,0 \mathrm{KGy}$ & $4,17 \mathrm{aA}$ & $5,15 \mathrm{abB}$ & $4,64 \mathrm{abB}$ & $5,29 \mathrm{abAB}$ & $6,14 \mathrm{bAB}$ & $6,89 \mathrm{bB}$ & $7,81 \mathrm{bcC}$ & $8,29 \mathrm{bC}$ \\
$1,0 \mathrm{~kJ} / \mathrm{m}^{2}$ & $4,17 \mathrm{aA}$ & $4,99 \mathrm{aB}$ & $4,35 \mathrm{aAB}$ & $5,00 \mathrm{aA}$ & $5,55 \mathrm{aB}$ & $6,21 \mathrm{aB}$ & $7,05 \mathrm{aB}$ & $7,42 \mathrm{aB}$ \\
$2,0 \mathrm{~kJ} / \mathrm{m}^{2}$ & $4,17 \mathrm{aA}$ & $5,11 \mathrm{abB}$ & $4,6 \mathrm{abB}$ & $5,21 \mathrm{aAB}$ & $6,22 \mathrm{bAB}$ & $7,11 \mathrm{bB}$ & $7,62 \mathrm{bcB}$ & $8,31 \mathrm{bC}$ \\
\hline
\end{tabular}

Médias seguidas pela mesma letra maiúsculas na linha (dias) e minúsculas na coluna (tratamentos) não diferem entre si pelo teste de Tukey. 
Os frutos do tratamento controle diferiram dos demais, principalmente a partir do nono dia de avaliação, quando se observou redução dos teores de acidez titulável, alcançando média de 2,02\% de ácido ascórbico/100 g de polpa ao final de 21 dias de armazenamento. As dosagens e as formas de irradiação não diferiram entre si, evidenciando redução gradual a partir do décimo quinto dia e alcançando valores médios variando entre 2,32 e 2,36\% de ácido ascórbico/100 g de polpa ao fim de 21 dias de armazenamento, fato este importante, pois revela o efeito positivo da irradiação na manutenção deste parâmetro (Tabela 2).

As médias verificadas variaram do início ao fim do experimento entre 2,89 e 2,08\% de ácido ascórbico/100 g de polpa concordando com os valores descritos por Oliveira et al. (2013) quando avaliaram a influência da atmosfera modificada e irradiação na vida de prateleira de camu-camu verificando valores entre 2,99 e 2,02 ao longo de 25 dias de armazenamento refrigerado.

Tibola et al. (2007), trabalhando com morangos, verificaram que a acidez titulável diferiu significativamente nos morangos tratados com UVC em comparação às frutas do tratamento controle. O mesmo foi verificado por Milagres et al. (2012), que observaram o efeito positivo das doses de radiação gama sobre o tratamento controle no armazenamento refrigerado de pimentas.

$\mathrm{Na}$ tabela 3, observa-se que os valores de $\mathrm{pH}$ do camu-camu se mostraram-se estáveis, nos tratamentos aplicados e em relação ao tempo de armazenamento, com valor mínimo de 2,09 e máximo de 2,93. Avaliando a conservação de camu-camu sob influência do estádio de maturação e das doses de radiação, Silva et al. (2013) encontraram valores semelhantes aos verificados nesta pesquisa, oscilando entre 2,19 e 2,79. $\mathrm{O}$ baixo valor verificado para o $\mathrm{pH}$ dos frutos de camucamu é importante, sendo um fator limitante para o crescimento de bactérias patogênicas, além de favorecer a estabilidade do ácido ascórbico em $\mathrm{pH}$ ácido.

Maeda et al. (2007) e Santos et al. (2010) também observaram redução nos valores de $\mathrm{pH}$ com o tempo de armazenamento em camu-camu e mangas sob irradiação sem diferença significativa quando comparados ao grupo controle.

Tabela 2. Valores médios pelo teste de Tukey nos valores de acidez titulável (\% de ácido ascórbico/100 g de polpa) em frutos de camu-camu tratados com diferentes fontes de radiação e armazenados sob refrigeração por 21 dias.

\begin{tabular}{|c|c|c|c|c|c|c|c|c|}
\hline \multirow{2}{*}{ Tratamentos } & \multicolumn{8}{|c|}{ Dias de análise } \\
\hline & 0 & 3 & 6 & 9 & 12 & 15 & 18 & 21 \\
\hline Controle & $2,99 \mathrm{aA}$ & $2,87 \mathrm{aA}$ & $2,93 \mathrm{aA}$ & $3,03 \mathrm{bB}$ & $2,57 \mathrm{bC}$ & $2,31 \mathrm{bC}$ & $2,19 \mathrm{bD}$ & $2,02 \mathrm{bD}$ \\
\hline $1,0 \mathrm{KGy}$ & $2,99 \mathrm{aA}$ & $2,93 \mathrm{aA}$ & $2,84 \mathrm{aA}$ & $2,89 \mathrm{aA}$ & $3,00 \mathrm{aB}$ & $2,76 \mathrm{aB}$ & $2,46 \mathrm{aBC}$ & $2,36 \mathrm{aC}$ \\
\hline 2,0 KGy & $2,99 \mathrm{aA}$ & $2,91 \mathrm{aA}$ & $2,79 \mathrm{aA}$ & $2,93 \mathrm{aAB}$ & $3,03 \mathrm{aB}$ & $2,71 \mathrm{aB}$ & $2,52 \mathrm{aBC}$ & $2,32 \mathrm{aC}$ \\
\hline $1,0 \mathrm{~kJ} / \mathrm{m}^{2}$ & $2,99 \mathrm{aA}$ & $2,94 \mathrm{aA}$ & $2,85 \mathrm{aA}$ & $2,92 \mathrm{aA}$ & $2,99 \mathrm{aB}$ & $2,74 \mathrm{aB}$ & $2,48 \mathrm{aBC}$ & $2,36 \mathrm{aC}$ \\
\hline $2,0 \mathrm{~kJ} / \mathrm{m}^{2}$ & $2,99 \mathrm{aA}$ & $2,89 \mathrm{aA}$ & $2,81 \mathrm{aA}$ & $2,95 \mathrm{aAB}$ & $3,08 \mathrm{aB}$ & $2,81 \mathrm{aB}$ & $2,41 \mathrm{aBC}$ & $2,33 \mathrm{aC}$ \\
\hline
\end{tabular}

Tabela 3. Valores médios de $\mathrm{pH}$ em frutos de camu-camu tratados com diferentes fontes de radiação e armazenados sob refrigeração por 21 dias

\begin{tabular}{ccccccccc}
\hline \multirow{2}{*}{ Tratamentos } & \multicolumn{10}{c}{ Dias de análise } \\
\cline { 3 - 9 } & 0 & 3 & 6 & 9 & 12 & 15 & 18 & 21 \\
\cline { 3 - 8 } Controle & $2,09 \mathrm{aA}$ & $2,29 \mathrm{aAB}$ & $2,48 \mathrm{aB}$ & $2,74 \mathrm{aB}$ & $2,85 \mathrm{aB}$ & $2,59 \mathrm{aC}$ & $2,27 \mathrm{aD}$ & $2,18 \mathrm{aD}$ \\
$1,0 \mathrm{KGy}$ & $2,09 \mathrm{aA}$ & $2,25 \mathrm{aAB}$ & $2,42 \mathrm{aB}$ & $2,71 \mathrm{aB}$ & $2,93 \mathrm{aBC}$ & $2,67 \mathrm{aC}$ & $2,34 \mathrm{aD}$ & $2,22 \mathrm{aD}$ \\
$2,0 \mathrm{KGy}$ & $2,09 \mathrm{aA}$ & $2,25 \mathrm{aAB}$ & $2,48 \mathrm{aB}$ & $2,75 \mathrm{aB}$ & $2,81 \mathrm{aB}$ & $2,61 \mathrm{aC}$ & $2,29 \mathrm{aD}$ & $2,19 \mathrm{aD}$ \\
$1,0 \mathrm{~kJ} / \mathrm{m}^{2}$ & $2,09 \mathrm{aA}$ & $2,21 \mathrm{aAB}$ & $2,4 \mathrm{aB}$ & $2,70 \mathrm{aB}$ & $2,93 \mathrm{aBC}$ & $2,69 \mathrm{aC}$ & $2,36 \mathrm{aD}$ & $2,21 \mathrm{aD}$ \\
$2,0 \mathrm{~kJ} / \mathrm{m}^{2}$ & $2,09 \mathrm{aA}$ & $2,23 \mathrm{aAB}$ & $2,51 \mathrm{aB}$ & $2,76 \mathrm{aB}$ & $2,87 \mathrm{aB}$ & $2,58 \mathrm{aC}$ & $2,29 \mathrm{aD}$ & $2,19 \mathrm{aD}$ \\
\hline
\end{tabular}

Médias seguidas pela mesma letra maiúsculas na linha (dias) e minúsculas na coluna (tratamentos) não diferem entre si pelo teste de Tukey. 
Com relação à variável vitamina $\mathrm{C}$ (Tabela 4), os valores apresentaram diferenças entre os tratamentos $\mathrm{e}$ os tempos de armazenamento; de modo geral, o uso da irradiação manteve os níveis de acidez através da conservação proporcionada sobre os frutos. Nota-se uma redução nos valores do primeiro ao último dia de armazenamento, de forma mais expressiva a partir do nono dia para os frutos do tratamento controle; no décimo quinto dia para os frutos irradiados a 2,0 de $\mathrm{KGy}$ e $\mathrm{KJ} / \mathrm{m}^{2}$ e a partir do décimo oitavo dia para aqueles tratados sobre a dosagem de 1,0 de radiação gama e ultravioleta. (Tabela 4).

No fim do experimento, todos os tratamentos apresentaram redução com valor mais expressivo nos frutos do tratamento controle cuja média foi de 0,869 mg de ácido ascórbico por $100 \mathrm{~g}$ de polpa. As diferentes doses e fontes de irradiação não diferiram no último dia de avaliação apresentando médias oscilando entre 1.171 e 1.183 (Tabela 4). Os valores iniciais para o teor de vitamina $C$ verificados nos frutos foram de $1.922 \mathrm{mg}$ de ácido ascórbico por $100 \mathrm{~g}$ de polpa, sendo superiores aos valores relatados por Rufino et al., (2010) cujas médias corresponderam a 1.721 e 1.882 mg de ácido ascórbico por $100 \mathrm{~g}$ de polpa. Essa variação nos teores de vitamina $\mathrm{C}$ nos alimentos é variável de acordo com a região de cultivo, clima, época de colheita, mesmo sendo da mesma variedade.

Os valores médios dos carotenóides nos frutos de camu-camu apresentaram reduções com o tempo de armazenamento com médias oscilando do primeiro ao último dia de armazenamento entre 312,5 e 86,13 mg/100 g de polpa (Tabela 5). Maeda et al., (2007) avaliando frutos de camu-camu, notaram redução nos teores de carotenóides independente do estádio de maturação, atingindo médias de $70 \mathrm{mg} / 100 \mathrm{~g}$ de polpa ao fim do período de armazenamento.

Nota-se em todos os tratamentos com irradiação certa estabilidade nos teores de carotenóides até o décimo quinto dia com posterior redução, até o fim do armazenamento. Os frutos tratados nas dosagens de 2,0 KGy de irradiação gama e 2,0 e $\mathrm{KJ} / \mathrm{m}^{2}$ ultravioleta, apresentaram redução mais lenta a partir desse período, diferindo significativamente dos demais. O tratamento controle apresentou redução no teor de carotenóides ao longo de todo o tempo de armazenamento, principalmente, a partir do nono dia de avaliação (Tabela 5).

Tabela 4. Valores médios de vitamina C (mg de ácido ascórbico por $100 \mathrm{~g}$ de polpa) em frutos de camu-camu tratados com diferentes fontes de radiação e armazenados sob refrigeração por 21 dias.

\begin{tabular}{ccccccccc}
\hline \multirow{2}{*}{ Tratamentos } & \multicolumn{7}{c}{ Dias de análise } \\
\cline { 2 - 7 } & 0 & 3 & 6 & 9 & 12 & 15 & 18 & 21 \\
\cline { 3 - 8 } Controle & $1,922 \mathrm{aA}$ & $1,891 \mathrm{aA}$ & $1,785 \mathrm{bAB}$ & $1,623 \mathrm{bAB}$ & $1,436 \mathrm{cB}$ & $1,282 \mathrm{cC}$ & $1,109 \mathrm{cC}$ & $0,869 \mathrm{bC}$ \\
$1,0 \mathrm{KGy}$ & $1,922 \mathrm{aA}$ & $1,907 \mathrm{aA}$ & $1,872 \mathrm{aA}$ & $1,773 \mathrm{aA}$ & $1,687 \mathrm{aA}$ & $1,593 \mathrm{aA}$ & $1,422 \mathrm{aA}$ & $1,176 \mathrm{aB}$ \\
$2,0 \mathrm{KGy}$ & $1,922 \mathrm{aA}$ & $1,905 \mathrm{aA}$ & $1,786 \mathrm{abA}$ & $1,702 \mathrm{abA}$ & $1,621 \mathrm{bA}$ & $1,406 \mathrm{bB}$ & $1,213 \mathrm{bAB}$ & $1,183 \mathrm{aB}$ \\
$1,0 \mathrm{~kJ} / \mathrm{m}^{2}$ & $1,922 \mathrm{aA}$ & $1,909 \mathrm{aA}$ & $1,814 \mathrm{aA}$ & $1,771 \mathrm{aA}$ & $1,688 \mathrm{aA}$ & $1,599 \mathrm{aB}$ & $1,414 \mathrm{aB}$ & $1,180 \mathrm{aB}$ \\
$2,0 \mathrm{~kJ} / \mathrm{m}^{2}$ & $1,922 \mathrm{aA}$ & $1,903 \mathrm{aA}$ & $1,777 \mathrm{abA}$ & $1,708 \mathrm{abA}$ & $1,617 \mathrm{bA}$ & $1,409 \mathrm{bB}$ & $1,205 \mathrm{bAB}$ & $1,175 \mathrm{aB}$ \\
\hline
\end{tabular}

$\mathrm{CV}(\%)=0,87$

Médias seguidas pela mesma letra maiúsculas na linha (dias) e minúsculas na coluna (tratamentos) não diferem entre si pelo teste de Tukey.

Tabela 5. Valores médios sobre de carotenóides $(\mathrm{mg} / 100 \mathrm{~g}$ de polpa) em frutos de camu-camu tratados com diferentes fontes de irradiação e armazenados sob refrigeração por 21 dias.

\begin{tabular}{|c|c|c|c|c|c|c|c|c|}
\hline \multirow{2}{*}{ Tratamentos } & \multicolumn{8}{|c|}{ Dias de análise } \\
\hline & 0 & 3 & 6 & 9 & 12 & 15 & 18 & 21 \\
\hline Controle & $312,5 \mathrm{aA}$ & $285,9 \mathrm{bB}$ & $242,4 \mathrm{bB}$ & $214,8 \mathrm{bB}$ & $162,9 \mathrm{bC}$ & $121,1 \mathrm{bD}$ & $100,5 \mathrm{cD}$ & $86,13 \mathrm{cD}$ \\
\hline 1,0 KGy & $312,5 \mathrm{aA}$ & $300,2 \mathrm{aA}$ & $282,5 \mathrm{aAB}$ & $265,5 \mathrm{aB}$ & $229,3 \mathrm{aB}$ & $202,1 \mathrm{aBC}$ & $174,3 \mathrm{abC}$ & $155,3 \mathrm{abC}$ \\
\hline 2,0 KGy & $312,5 \mathrm{aA}$ & $300,1 \mathrm{aA}$ & $281,4 \mathrm{aAB}$ & $266,4 \mathrm{aB}$ & $234,8 \mathrm{aB}$ & $212,7 \mathrm{aBC}$ & $193,6 \mathrm{aC}$ & $176,5 \mathrm{aC}$ \\
\hline $1,0 \mathrm{~kJ} / \mathrm{m}^{2}$ & $312,5 \mathrm{aA}$ & $300,3 \mathrm{aA}$ & $280,3 \mathrm{aAB}$ & $265,7 \mathrm{aB}$ & $227,4 \mathrm{aB}$ & $209,6 \mathrm{aBC}$ & $178,4 \mathrm{abC}$ & $151,8 \mathrm{abC}$ \\
\hline $2,0 \mathrm{~kJ} / \mathrm{m}^{2}$ & $312,5 \mathrm{aA}$ & $300,2 \mathrm{aA}$ & $282,6 \mathrm{aAB}$ & $267,9 \mathrm{aB}$ & $237,6 \mathrm{aB}$ & $210,6 \mathrm{aBC}$ & $196,7 \mathrm{aC}$ & $171,5 \mathrm{aC}$ \\
\hline
\end{tabular}

Médias seguidas pela mesma letra maiúsculas na linha (dias) e minúsculas na coluna (tratamentos) não diferem entre si pelo teste de Tukey. 
Essa manutenção do teor de carotenoides pode ter sido estimulada possivelmente pelos efeitos da irradiação que ativou os mecanismos de defesa do fruto, sendo estes mecanismos responsáveis por vários fenômenos nos frutos; um deles é o responsável por promover a indução do metabolismo de carotenóides (ALOTHMAN et al., 2009; LIU et al., 2009). Campos e Vieites (2009), inferem em seu trabalho com tomates, que as maiores concentrações de radiação gama (2,0 e 3,0 de KGy) proporcionaram as maiores médias de carotenóides ao longo do armazenamento, corroborando com os resultados observados neste estudo, cuja maior média de carotenóides foi encontrada nas maiores dosagens, independente da fonte de irradiação.

Os dados da taxa respiratória nos diferentes tratamentos são apresentados na Figura 3; de maneira geral observa-se um padrão climatérico de desenvolvimento, assim como sugerido por Corrêa et al. (2007) onde após a colheita dos frutos, a respiração torna-se o principal processo fisiológico utilizando suas próprias reservas para continuar o seu desenvolvimento.

Ao longo de todo o tempo de armazenamento observou-se um incremento na taxa respiratória dos frutos evidenciando diferentes picos respiratórios nos diferentes tratamentos aplicados. O tratamento controle apresentou maior média no décimo segundo dia de avaliação; 12,6 mLCO $\mathrm{Kg}^{-1} \mathrm{~h}^{-1}$ e apresentando valor de $8,54 \mathrm{mLCO}_{2} \mathrm{Kg}^{-1} \mathrm{~h}^{-1}$ no fim do experimento. Os tratamentos com dosagem de $1,0 \mathrm{e} \mathrm{KJ} / \mathrm{m}^{2}$ verificaram pico respiratório ao décimo oitavo dia com médias variando entre $15,8 \mathrm{mLCO}_{2} \mathrm{Kg}^{-1} \mathrm{~h}^{-1}$ e $16,1 \mathrm{mLCO}_{2} \mathrm{Kg}$ ${ }^{1} \mathrm{~h}^{-1}$ para radiação gama e ultravioleta, respectivamente (Figura 3). Esse pico respiratório apresentado nos diferentes tratamentos é comum nos frutos climatéricos; logo após, como evidência da fase pós-climatérica ocorre o decréscimo da taxa respiratória que corresponde ao início da senescência dos frutos (CHITARRA e CHITARRA, 2007).

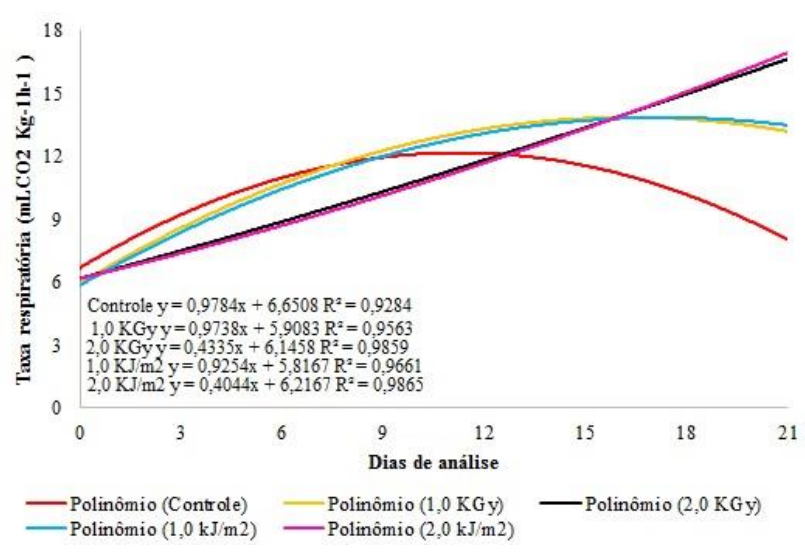

Figura 3. Taxa respiratória $\left(\mathrm{mLCO}_{2} \mathrm{Kg}^{-1} \mathrm{~h}^{-1}\right)$ em frutos de camu-camu tratados com diferentes fontes de radiação e armazenados sob refrigeração por 21 dias.
As dosagens de 2,0 KGy e $\mathrm{KJ} / \mathrm{m}^{2}$ de radiação gama e ultravioleta apresentaram certa estabilidade ao longo de todo o tempo de armazenamento, diferindo estatisticamente entre si. Observou-se um incremento lento na taxa respiratória dos frutos até o décimo oitavo dia atingindo média de 14,3 $\mathrm{mLCO}_{2} \mathrm{Kg}^{-1} \mathrm{~h}^{-1}$ e 14,9 $\mathrm{mLCO}_{2} \mathrm{Kg}^{-1} \mathrm{~h}^{-1}$ com aumento mais significativo no último dia de avaliação, quando se verificou médias de 16,9 e $16,4 \mathrm{mLCO}_{2} \mathrm{Kg}^{-1} \mathrm{~h}^{-1}$ para radiação gama e ultravioleta respectivamente (Figura 3 ).

\section{Conclusões}

As diferentes fontes de radiação (gama e ultravioleta) revelaram-se eficientes no aumento da vida de prateleira do camu-camu in natura.

Os tratamentos com irradiação não comprometeram as características físico-químicas e permitiram uma vida de prateleira por 18,15 e 9 dias para as doses de 2,0 e 1,0 de (irradiação gama/ultravioleta) e controle respectivamente, em ambiente refrigerado, sendo a dose de 2,0 KGy e $\mathrm{KJ} / \mathrm{m}^{2}$ mais eficiente em gerar estresse sobre a fisiologia dos frutos, preservando sua qualidade.

\section{Referências Bibliográficas}

ALAM KHAN, K.; ABRAHEM, M. Effect of irradiation on quality of spices. International Food Research Journal, Selangor-Malaysia, v.17, n. 1, p. 825-836, 2010.

ALOTHMAN, M.; BHAT, R.; KARIM, A. A. UV radiationinduced changes of antioxidante capacity of fresh-cut tropical fruits. Innovative Food Science and Emerging Technologies, Penang, Malaysia, v. 10, n. 2 p. 512-516, 2009.

ALVES, R. E., FILGUEIRAS, H. A. C., MOURA, C. F. H., ARAÚJO, N. C. C., ALMEIDA, A. S. Camu-Camu (Myrciaria dubia Mc Vaugh): a rich natural source of vitamin C. Proceedings of the Interamerican Society for Tropical Horticulture, Leuven, Belgium, v. 46, n. 1, p. 11-13, 2002.

AOAC. ASSOCIATION OF OFFICIAL ANALYTICAL CHEMISTS. Offifial methods of the association of the agricultural Chemists. $17^{\text {th }}$ ed. Waschington-USA: AOAC, 2007. $1410 \mathrm{p}$

BLEINROTH, E. W., ZUCCHINI, A. G.; POMPEO, R. M. Determinação das características físicas e mecânicas de variedade de abacate e sua conservação pelo frio. Coletânea ITAL, Campinas-SP, v. 7, n. 1, p. 29-81, 1976.

CAMPOS, A. J.; VIETES, R. L. Ultravioleta (UVC) na conservação da qualidade de tomate. Revista Eletrônica de Agronomia, Garça-SP, v. 16, n. 2, p. 20-26, 2009.

CAMPOS, A. J.., VIEITES, R. L. Conservação refrigerada de uva 'Itália' com utilização da irradiação. Revista Científica Eletrônica de Agronomia, Garça-SP, v. 17, n. 1, p. 31-43, 2010. 
CAMPOS, A. J., FUJITA, E., NEVES, L. C., VIEITES, R. L., CHAGAS, E, A., Radiação gama e atmosfera modificada passiva na qualidade de goiabas 'Pedro Sato'. Revista Brasileira de Fruticultura, Jaboticabal-SP, Volume Especial, p. 350-356, 2011.

CHITARRA, M. I. F, CHITARRA, A. B. Pós-colheita de frutos e hortaliças: fisiologia e manuseio. 2. ed. Lavras-MG: Editora UFLA, 2005.

CHITARRA, M. I. F.; CHITARRA, A. B. Processamento mínimo de alface. In: MORETTI, C. L. (Ed.). Manual de processamento mínimo de frutas e hortaliças. Brasília-DF: Embrapa Hortaliças e SEBRAE, 2007. Cap. 16, p. 301-341.

CORRÊA, M. O. G.; PINTO, D. D.; ONO, E. O. Análise da atividade respiratória em frutos de jabuticabeira. Revista Brasileira de Biociências, Porto Alegre-RS, v. 5, n. 2, p. 831833, 2007.

DAIUTO, E. R., VIEITES, R. L., TREMOCOLDI, M. A., RUSSO, V. C. Taxa respiratória de abacate 'hass' submetido a diferentes tratamentos físicos. Revista Iberoamericana de Tecnología Postcosecha. Hermosillo, México, v, 10, n. 2, p. 101-109, 2010.

FAN, X., NIEMIRA, B.A., PRAKASH, A. Irradiation of fresh fruits and vegetables. Food Technology, Chicago-USA, v. 3, n. 2 , p. $36-43.2012$.

FRANÇOSO, I. L. T., COUTO, M. A. L., CANNIATTIBRAZACA, S. G., ARTHUR, V. Alterações físico-químicas em morangos (Fragaria anassa Duch.) irradiados e armazenados. Ciência e Tecnologia de Alimentos, Campinas-SP, v. 28, n. 3, p. 614-619, 2008.

GUIMARÃES, I. C.; MENEZES, E. G. T.; ABREU, P. S.; RODRIGUES, A. C.; BORGES, P. R. S.; BATISTA, L. R.; CIRILO, M. A.; LIMA, L. C. O. Physicochemical and microbiological quality of raspberries (Rubus idaeus) treated with different doses of gamma irradiation. Food Science and Technology, Campinas-SP, v. 33, p. 316-322, 2013.

HIGBY, W. K. A simplified method for determination of some the carotenoid distribution in natural and carotenefortified orange juice. Journal of Food Science, Chicago-USA, v. 27, n. 1, p. 42-49, 1962.

IAL. INSTITUTO ADOLFO LUTZ. Métodos físicoquímicos para análise de alimentos. 4. ed. São Paulo-SP: Instituto Adolfo Lutz, 2008. 1020 p.
LIU, L. H.; ZABARAS, D.; BENNETT, L. E.; AGUAS, P.; WOONTON, B. W. Effects of UVC, red light and sun light on the carotenoid content and physical qualities of tomatões during post-harvest storage. Food Chemistry, Issue-China, v. 115, n. 3, p. 495-500, 2009.

MAEDA, R. N, PANTOJA, L., YUYAMA, L. K. O., CHAAR, J. M. Estabilidade de ácido ascórbico e antocianinas em néctar de camu-camu (Myrciaria dubia (H. B. K.) McVaugh). Ciência e Tecnologia de Alimentos, CampinasSP, v. 27, n. 2, p.313-316, 2007.

MILAGRES, R. C. R. M., POLESI, L. F., PIEDADE, J., CANNIATTI-BRAZACA, S. G., SPOTO, M. H. F., WALDER, J. M. M. Aplicação da radiação gama associada a diferentes temperaturas para conservação de pimenta (Capsicum baccatum var. pendulum) in natura. Revista Alimentos e Nutrição, Araraquara-SP, v. 23, n. 2, p. 223-233, 2012

OLIVEIRA, J.; CASTELUCCI, A. C. L.; SILVA, P. P. M.; SILVA, G. M.; SPOTO, M. H. F. Polpa de camu-camu (Myrciaria dubia) submetida à radiação gama. Acta Agronómica. Palmira-Colômbia, v. 62, n. 1, p. 7-12, 2013.

RUFINO, M. S. M. ALVES, R. E.; BRITO, E. S.; PÉREZJIMÉNEZ, J.; SAURA-CALIXTO, F.; MANCINI-FILHO, J. Bioactive compounds and antioxidant capacities nontraditional tropical fruits from Brazil. Food Chemistry, IssueChina, v.121, n. 4, p. 996-1002, 2010

SANTOS, A. M. G. OLIVEIRA, S. M. A.; SILVA, J. M., TERÃO, D. Podridão por Fusicoccum em mangas submetidas a baixas doses de radiação gama. Pesquisa Agropecuária Brasileira. Brasilia-DF, v. 45, n. 10, p. 1066-1072, 2010.

SILVA, M. A.; SOBRAL, P. J. A.; KIECKBUSCH, T. G. State diagrams of freeze-dried camu-camu (Myrciaria dubia (HBK) Mc Vaugh) pulp with and without maltodextrin addition. Journal of Food Engineering, Londres-UK, v. 77, n. 3 , p. 426-432, 2013.

TIBOLA, C. S.; MALGARIM, M. B.; ZAICOVSKI, C. B.; PEGORARO, C.; CERO, J.; FERRI, V. C. Luz ultravioleta na inibição de podridões pós-colheita de morangos (Fragaria ananassa, Duch.) 'Camarosa'. Revista Brasileira AgroCiência, Pelotas-RS, v.13, n.4, p.509- 512, 2007.

ZANATTA, C. F.; MERCADANTE, A. Z. Carotenoid composition from the Brazilian tropical fruit camu-camu (Myrciaria dubia). Food Chemistry, Issue-China, v. 101, n. 1, p.1526-1532, 2007. 